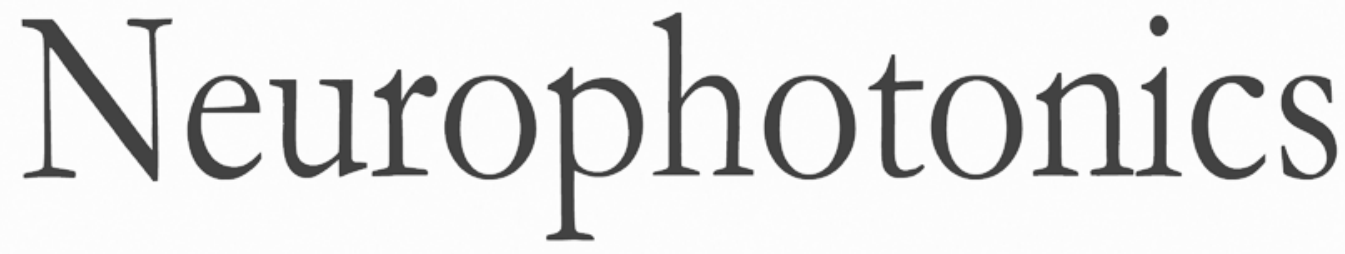

\title{
Theoretical analysis of low-power fast optogenetic control of firing of Chronos-expressing neurons
}

Sant Saran

Neha Gupta

Sukhdev Roy 


\title{
Theoretical analysis of low-power fast optogenetic control of firing of Chronos-expressing neurons
}

\author{
Sant Saran, ${ }^{a}$ Neha Gupta, ${ }^{b}$ and Sukhdev Roy ${ }^{b, *}$ \\ ${ }^{a}$ Dayalbagh Educational Institute, Department of Electrical Engineering, Agra, Uttar Pradesh, India \\ bDayalbagh Educational Institute, Department of Physics and Computer Science, Agra, Uttar Pradesh, India
}

\begin{abstract}
A detailed theoretical analysis of low-power, fast optogenetic control of firing of Chronos-expressing neurons has been presented. A three-state model for the Chronos photocycle has been formulated and incorporated in a fast-spiking interneuron circuit model. The effect of excitation wavelength, pulse irradiance, pulse width, and pulse frequency has been studied in detail and compared with ChR2. Theoretical simulations are in excellent agreement with recently reported experimental results and bring out additional interesting features. At very low irradiances $\left(0.005 \mathrm{~mW} / \mathrm{mm}^{2}\right)$, the plateau current in Chronos exhibits a maximum. At $0.05 \mathrm{~mW} / \mathrm{mm}^{2}$, the plateau current is 2 orders of magnitude smaller and saturates at longer pulse widths ( $700 \mathrm{~ms})$ compared to ChR2 ( $350 \mathrm{~ms})$. $I_{\text {peak }}$ in Chronos saturates at much shorter pulse widths (1775 pA at $1.5 \mathrm{~ms}$ and $5 \mathrm{~mW} / \mathrm{mm}^{2}$ ) than in ChR2. Spiking fidelity is also higher at lower irradiances and longer pulse widths compared to ChR2. Chronos exhibits an average maximal driven rate of over 200 spikes/s in response to 100 pulses/s stimuli, each of 1 -ms pulse-width, in the intensity range 0 to $200 \mathrm{~mW} / \mathrm{mm}^{2}$. The analysis is important to not only understand the photodynamics of Chronos and Chronos-expressing neurons but also to design opsins with optimized properties and perform precision experiments with required spatiotemporal resolution. ๑ 2018 Society of Photo-Optical Instrumentation Engineers (SPIE) [DOI: 10.1117/1.NPh.5.2.025009]
\end{abstract}

Keywords: optogenetics; Hodgkin-Huxley model; Wang-Buzsaki interneuron model; neural firing; channelrhodopsin; ChR2 and Chronos.

Paper 17138RR received Dec. 1, 2017; accepted for publication May 11, 2018; published online May 24, 2018.

\section{Introduction}

Recent years have witnessed tremendous research effort focused on the development of optogenetics as an innovative technology that has revolutionized manipulation of cellular behavior through light stimulation. ${ }^{1-3}$ It provides user-defined control of various cellular functions with highly precise spatiotemporal resolution and minimal invasiveness, both in vitro and in vivo, creating unprecedented opportunities for biomedical applications. ${ }^{4-12}$ Combination of this research tool with complementary technologies that include electrophysiology, activity imaging, and anatomical methods provides better understanding of physiology and behavior on acute time scales across cellular, circuit, and brain scales. ${ }^{13,14}$

Over the past decade, a wide range of optogenetic tools have been developed, primarily microbial rhodopsin pumps and channels. ${ }^{2}$ Light-triggered isomerization of retinal initiates a sequence of thermally driven conformational transitions in a photocycle and ultimately results in the movement of ions across the membrane..$^{2,15-19}$ When transgenically expressed in neurons, channelrhodopsins (ChRs) mediate light-dependent transport of cations into the cell, causing depolarization and stimulation of action potentials, or can selectively hyperpolarize the cell and inhibit action potentials, by either pumping protons out or pumping chloride into the cell. ${ }^{15-22}$

Intense research effort is at present directed to designing more effective opsins by tailoring their properties, including activation, unitary spectral conductance, retinal binding affinity,

*Address all correspondence to: Sukhdev Roy, E-mail: sukhdevroy@dei.ac.in faster temporal kinetics, light actuation sensitivity, spectral tuning, and protein stability. It is also very important to consider properties that strongly depend on target cells, such as expression level and membrane targeting. ${ }^{14}$ A major challenge is to overcome the inverse relationship between light sensitivity and kinetics to achieve low-power as well as ultrafast operation for higher spatiotemporal resolution.

Chronos, a blue and green light-sensitive opsin recently discovered through de novo sequencing of opsins from over 100 algal species, i.e., Stigeoclonium helveticum ChR, exhibits high light sensitivity and unprecedented kinetics, with a turnon of $2.3 \pm 0.3 \mathrm{~ms}$ and turn-off of $3.6 \pm 0.2 \mathrm{~ms}$. It is the fastest ChR reported to date. ${ }^{8}$ Chronos-mediated optical spiking replicates electrically driven spiking between 5 and $60 \mathrm{~Hz}$. Hence, Chronos represents an excellent general-use ChR that has been recently shown to offer tremendous advantages in central auditory optoprostheses, auditory brainstem implant performance, and sub-ms optogenetic control of a broad range of physiological firing patterns with two-photon holographic photoactivation. ${ }^{8,10,11,23}$

To design and control optogenetic devices and circuits, it is extremely important to also develop a theoretical understanding of the biophysical mechanism of the photosensitization agents and their behavior in cell. Hence, computational modeling is fundamental to understand the light-induced ionic transport in cell membrane, for not only cell physiology but also to develop new treatments. ${ }^{24-28}$ Although the photoresponse of Chronos and Chronos-expressing neurons has only been experimentally 
studied, it is also important to undertake a theoretical analysis to understand their photodynamics. ${ }^{8}$

The objective of this paper is to present a detailed theoretical analysis to (i) formulate an accurate model of the photocycle of Chronos, (ii) study the effect of various parameters that include irradiance, wavelength, pulse width, and pulse frequency, (iii) develop an integrated neuron circuit model to accurately simulate ultrafast firing in Chronos-expressing neurons, (iv) compare theoretical results with recently reported experimental results, and (v) derive optimized conditions for optogenetic stimulation of Chronos and Chronos-expressing neurons.

\section{Theoretical Model}

Photoexcitation of microbial rhodopsins leads to isomerization of the retinal moity around the $\mathrm{C}_{13}=\mathrm{C}_{14}$ bond, from all-trans to 13-cis, followed by thermally driven reaction that eventually restores the initial $\mathrm{C}_{13}=\mathrm{C}_{14}$ conformation in a photocycle. ${ }^{18,29-31}$ It has been experimentally shown that Chronos exhibits a faster photocurrent decay, which implies that it can be accurately modeled with a mono-exponential fit. ${ }^{8}$ This suggests an accurate description of its photocycle by a three-state model as shown in Fig. 1.

On optical excitation, we consider Chronos molecule to undergo a very fast transition from the closed sensitive state (C) to an open-channel excited state (O), which spontaneously turns into a closed desensitized state (D). Although, subsequently the ion channel is closed, the molecule is not ready to photoswitch again. After a recovery period, the molecule then transits back to $\mathrm{C}$. The $\mathrm{O}$ to $\mathrm{D}$ turn off time for $\mathrm{ChR} 2$ is $\sim 10$ to $400 \mathrm{~ms}$, whereas the turn off time for Chronos is only $3.6 \mathrm{~ms}^{8}{ }^{8}$ To describe the response of Chronos-expressing neurons to light stimuli, we present a model that combines the kinetics of the Chronos photocycle and a single-compartment, fast-spiking interneuron model as shown in Fig. 1.

The effect of the induced conductance on the neuron is determined with Hodgkin-Huxley $(\mathrm{H}-\mathrm{H})$ type elements. ${ }^{32}$ The inputs to the model are the membrane capacitance and level of ChR2 expression (i.e., the number of ChR2s per unit area) as the physical properties of the neuron cell and stimulation irradiance and timing as independent variables. We demonstrate the efficacy of the model by comparing with experimental results and use the model to investigate different stimulation profiles. We assume

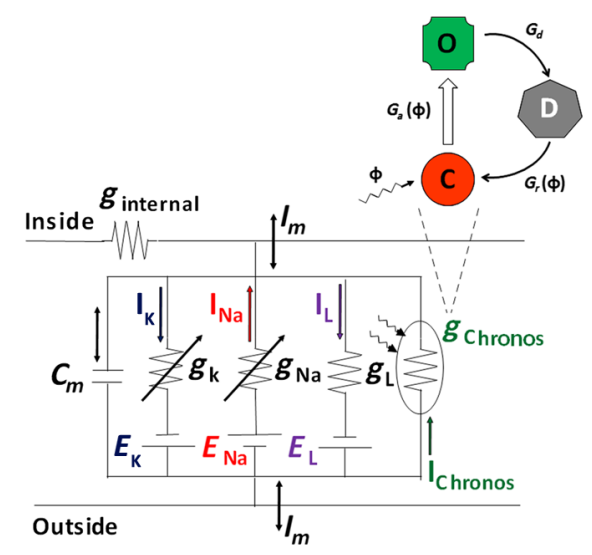

Fig. 1 Schematic of equivalent circuit diagram of Chronos-expressed neuron, where $C_{m}$ is the membrane potential, $I_{\mathrm{K}}, I_{\mathrm{Na}}$, and $I_{\mathrm{L}}$ are the ionic currents, $g_{\mathrm{K}}, g_{\mathrm{Na}}$, and $g_{\mathrm{L}}$ are the voltage-dependent conductances, and $g_{\text {Chronos }}$ is the light-dependent conductance governed by Chronos photocycle. that all light-sensitive ion-channel currents $(I)$ can be expressed in the classic form

$I=g(V-E)$

where $g$ is the channel conductance, $V$ is the membrane voltage, and $E$ is the reversal potential for the specific opsin type. We use a simplified empirical form for the channel conductance, introduced by Hodgkin and Huxley, ${ }^{28,32}$ expressing it as a product of a constant $\left(g_{o}\right.$, which is the maximum conductance at $V=-65 \mathrm{mV})$ and a numerical coefficient $(f>0)$. In general, $g=g(\phi, \lambda, V, T, \mathrm{pH}, t)=g_{0}(\lambda) \cdot f(\phi, V, T, \mathrm{pH}, t)$, where $\phi$ is the photon flux per unit area, $V$ is the membrane voltage, $T$ is the temperature, and $t$ is the time. We consider that the photocycle and membrane voltage dependencies can be separated as $g(\phi, \lambda, V, t)=g_{o}(\lambda) f_{\phi}(\phi, t) f_{v}(V)$, where $g_{o}(\lambda)$ is the maximum conductance at membrane voltage $V=-65 \mathrm{mV}$ at a particular wavelength, $f_{\phi}(\phi, t)$ is the normalized light-dependent function, and $f_{v}(V)$ is the voltage-dependent function. The voltage dependence of Chronos is derived from photocurrent recordings under constant irradiance and changing clamped voltages. We consider $f_{v}(V)=\left[v_{1} /(V-E)\right]\left\{1-\exp \left[-(V-E) / v_{o}\right]\right\}$, where $v_{o}, v_{1}$ are the fitting parameters along with $E$, the channel's reversal potential, and $f_{v}(V)=1$ at $-65 \mathrm{mV}$ (rest potential).

We introduce a three-state kinetic model for the photodynamics of Chronos, which consists of three functional states $\mathrm{C}, \mathrm{O}$, and $\mathrm{D}$. The light-mediated transition process from closed-state $\mathrm{C}$ to the open-state $\mathrm{O}$ is faster $\left(\tau_{\mathrm{on}} \sim 2.3 \mathrm{~ms}\right)$ than the transition from open-state $\mathrm{O}$ to the desensitized state $\mathrm{D}\left(\tau_{\text {off }} \sim 3.6 \mathrm{~ms}\right)$ and recovery from $\mathrm{D}$ to $\mathrm{C}\left(\tau_{r} \sim 50 \mathrm{~s}\right)$ at $\mathrm{pH}=7.3 .{ }^{8}$ Considering $\mathrm{C}$, $\mathrm{O}$, and $\mathrm{D}$ to denote the fraction of Chronos molecules in each of the three states at any given instant of time, the transition rates for the kinetics can be described by the following set of equations: ${ }^{28}$

$\dot{C}=G_{r}(\phi) D-G_{a}(\phi) C$,

$\dot{O}=G_{a}(\phi) C-G_{d} O$

$\dot{D}=G_{d} O-G_{r}(\phi) D$,

where $C+O+D=1 . G_{a}, G_{d}$, and $G_{r}$ are the rate constants for $C \rightarrow O, O \rightarrow D$, and $D \rightarrow C$ transitions, respectively, determined from the experimental data and defined as $G_{a}(\phi)=$ $k_{a} \phi^{p} /\left(\phi^{p}+\phi_{m}^{p}\right), G_{d}=1 /\left(\tau_{\mathrm{off}}\right)$, and $G_{r}(\phi)=\frac{k_{r} \phi^{q}}{\phi^{q}+\phi_{m}^{q}}+G_{r 0}$, where $G_{r 0}$ is estimated by simulating two long light pulses with varying interpulse interval $t_{\mathrm{IPI}}(0$ to $250 \mathrm{~ms})$ and voltage clamp at $-65 \mathrm{mV}$ and fit an appropriate exponential of the form $I_{\text {peak }}(t)=I_{\text {peak } 0}-a \cdot \exp \left(-G_{r 0} \cdot t_{\mathrm{IPI}}\right)$, to the experimental data. ${ }^{8}$ $I_{\text {peak } 0}$ is the initial peak photocurrent and $a$ is a constant fitting parameter. Since the three-state model assumes only one open state $(\mathrm{O})$, the normalized light-dependent function for Chronos $f_{\phi}(\phi)=O$. The photon flux per unit area $\phi=\lambda I / h c$, where $\lambda$ is the wavelength, $I$ is irradiance, $h$ is Planck's constant, $c$ is the speed of light in vacuum, and $\phi_{m}$ is the fitting parameter of flux dependence, as given by Evans et al. ${ }^{28}$ We employ empirical estimation to determine other model parameters $\left\{\phi_{m}, p, q\right.$, $k_{a}$, and $k_{r}$ \} (Table 1$)^{8,24}$.

Neural response to optostimulation is evaluated using a single-compartment, fast-spiking Wang-Buzsaki (W-B) 
Table 1 Opsin model parameters. ${ }^{8,24}$

\begin{tabular}{lcc} 
Parameter & Chronos & ChR2 \\
\hline$G_{d}\left(\mathrm{~ms}^{-1}\right)$ & 0.2778 & 0.0909 \\
$G_{r 0}\left(\mathrm{~ms}^{-1}\right)$ & $2 \times 10^{-5}$ & 0.0061 \\
$g_{o}\left(\mathrm{mS} \mathrm{mm}^{-2}\right)$ & $4.068 \times 10^{-8}$ & $1.1406 \times 10^{-8}$ \\
$\phi_{\mathrm{m}}\left(\mathrm{ph} \cdot \mathrm{mm}^{-2} \mathrm{~s}^{-1}\right)$ & $7.7 \times 10^{17}$ & $7.7 \times 10^{17}$ \\
$k_{a}\left(\mathrm{~ms}^{-1}\right)$ & 93.25 & 93.25 \\
$k_{r}\left(\mathrm{~ms}^{-1}\right)$ & 0.01 & 0.01 \\
$p$ & 1 & 1 \\
$q$ & 1 & 1 \\
$E\left(\mathrm{mV}^{2}\right)$ & 0 & 0 \\
$f_{V}(V)$ & 1 & 1 \\
$V(\mathrm{mV})$ & -65 & -65 \\
$\lambda(\mathrm{nm})$ & 470 & 470 \\
$\phi\left(\mathrm{ph} . \mathrm{mm}^{-2} \mathrm{~s}^{-1}\right)$ & $1.0002 \times 10^{16}$ & $1.0002 \times 10^{16}$ \\
$\Delta t(\mathrm{~ms})$ & 5 & 5 \\
\hline & &
\end{tabular}

interneuron model. ${ }^{33}$ These interneurons have the ability to fire repetitive spikes at high frequencies. With fast kinetics of inactivation $(h)$ of $I_{\mathrm{Na}}$, the activation $(n)$ of $I_{\mathrm{K}}$, and relatively high threshold of $I_{\mathrm{K}}$, the interneuron model displays a large range of repetitive spiking frequencies in response to a constant injected current. The W-B model with the addition of light-dependent Chronos ion-channel current $\left(I_{\text {Chronos }}\right)$ can be expressed as a system of differential equations of the form

$C_{\mathrm{m}} \dot{V}=I_{D C}-I_{g}-I_{\text {Chronos }}$,

where $C_{\mathrm{m}}$ is the membrane capacitance, $V$ is the membrane voltage, and $I_{\mathrm{DC}}$ is the constant DC bias current that controls the excitability of the neuron. $I_{g}$ is the sum of the membrane ion-channel currents, $I_{\mathrm{Na}}\left(\mathrm{Na}^{+}\right.$current), $I_{\mathrm{K}}\left(\mathrm{K}^{+}\right.$current), and $I_{\mathrm{L}} \quad$ (leakage current) given as $I_{\mathrm{Na}}=g_{\mathrm{Na}} m_{\infty}^{3} h\left(V-E_{\mathrm{Na}}\right)$, $I_{\mathrm{K}}=g_{\mathrm{K}} n^{4}\left(V-E_{\mathrm{K}}\right)$, and $I_{\mathrm{L}}=g_{\mathrm{L}}\left(V-E_{\mathrm{L}}\right)$, where $g_{\mathrm{Na}}, g_{\mathrm{K}}$,

Table 2 Rate functions. ${ }^{26,33}$

\begin{tabular}{lc} 
Parameter & Rate function \\
\hline$\alpha_{m}(V)$ & $\frac{-0.1(V+35)}{\exp [-0.1(V+35)]-1}$ \\
$\beta_{m}(V)$ & $4 \exp \left[\frac{-(V+60)}{18}\right]$ \\
$\alpha_{h}(V)$ & $0.07 \exp \left[\frac{-(V+58)}{20}\right]$ \\
$\beta_{h}(V)$ & $\frac{1}{\exp [-0.1(V+28)]+1}$ \\
$\alpha_{n}(V)$ & $\frac{-0.01(V+34)}{\exp [-0.1(V+34)]-1}$ \\
$\beta_{n}(V)$ & $0.125 \exp \left[\frac{-(V+44)}{80}\right]$ \\
\hline
\end{tabular}

Table 3 Neuron model parameters. ${ }^{26,33}$

\begin{tabular}{lc} 
Parameter & Numeric value \\
\hline$E_{\mathrm{Na}}(\mathrm{mV})$ & 55 \\
$E_{\mathrm{K}}(\mathrm{mV})$ & -90 \\
$E_{\mathrm{L}}(\mathrm{mV})$ & -65 \\
$g_{\mathrm{Na}}\left(\mathrm{mS} \mathrm{cm}^{-2}\right)$ & 35 \\
$g_{\mathrm{K}}\left(\mathrm{mS} \mathrm{cm}^{-2}\right)$ & 9 \\
$g_{\mathrm{L}}\left(\mathrm{mS} \mathrm{cm}^{-2}\right)$ & 0.1 \\
$C_{m}\left(\mu \mathrm{F} \mathrm{cm}^{-2}\right)$ & 1 \\
$\varphi$ & 5 \\
$I_{\mathrm{DC}}\left(\mu \mathrm{A} \mathrm{cm}^{-2}\right)$ & -0.51 \\
$V(\mathrm{mV})$ & -65 \\
\hline
\end{tabular}

and $g_{\mathrm{L}}$ are the conductances for the $\mathrm{Na}, \mathrm{K}$, and leakage ions, respectively, $h$ is the inactivation variable, $n$ and $m_{\infty}$ are the activation variables that obey the first-order kinetics, $\dot{h}=$ $\varphi\left[\alpha_{h}(1-h)-\beta_{h} h\right], \quad \dot{n}=\varphi\left[\alpha_{n}(1-n)-\beta_{n} n\right], \quad$ and $\quad m_{\infty}=$ $\alpha_{m} /\left(\alpha_{m}+\beta_{m}\right)$, respectively. The functional forms of membrane voltage-dependent rate constants $\left(\alpha_{m}, \alpha_{h}, \alpha_{n}, \beta_{m}, \beta_{h}\right.$, and $\left.\beta_{n}\right)$ are given in Table 2 , and the numerical values of model parameters $\left(E_{\mathrm{Na}}, E_{\mathrm{K}}, E_{\mathrm{L}}, g_{\mathrm{Na}}, g_{\mathrm{K}}, g_{\mathrm{L}}, C_{m}, \varphi, I_{\mathrm{DC}}\right.$, and $\left.V\right)$ are given in Table $3{ }^{26,33}$ All the simulations were performed using the fourth-order Runge-Kutta method with a time step of 0.05 ms implemented in MATLAB ${ }^{\text {TM }}$ R2013b by suitably modifying codes available at ModelDB.

\section{Results}

The photoresponse of Chronos and ChR2 has been studied through numerical simulations using Eqs. (1)-(5), considering reported experimental parameters in Table 1. The variation of photocurrent with time on excitation with 5-ms pulses at $I=4.23 \mathrm{~mW} / \mathrm{mm}^{2}$, at blue $(470 \mathrm{~nm})$, and green $(530 \mathrm{~nm})$ wavelengths is shown in Fig. 2(a), for $g_{0}=4.068 \times 10^{-8}$ and $3.363 \times 10^{-8} \mathrm{mS} \mathrm{mm}^{-2}$, respectively. As is evident, the photocurrent generated at $470 \mathrm{~nm}(\sim 1700 \mathrm{pA})$ is greater than at $530 \mathrm{~nm}(\sim 1453 \mathrm{pA})$, as Chronos is more sensitive to blue wavelength. ${ }^{8}$ These theoretical values match with the reported experimental values. ${ }^{8}$ At $470 \mathrm{~nm}$, the time to peak $t_{\text {peak }}=$ $1.55 \mathrm{~ms}$, while at $530 \mathrm{~nm}$ it is $1.4 \mathrm{~ms}$. At a holding potential of $-65 \mathrm{mV}$, cations flow into the cell interior and a negative current is recorded, a process that gets reversed when the potential polarity is reversed. The variation is more clearly understood on studying the population dynamics shown in the inset of Fig. 2(a). Absorption of blue photons triggers the Chronos photocycle. The molecules switch from the closed C-state to the open cation-selective ion-channel O-state.

This channel opens rapidly after absorption of a photon to generate a large permeability for monovalent and divalent cations. As the turn-on and turn-off time of Chronos are small and flux dependent, the population of the O-state attains a maximum value, while the light is still on and the molecules relax to the $\mathrm{D}$-state, resulting in increase in its population, which is retained 


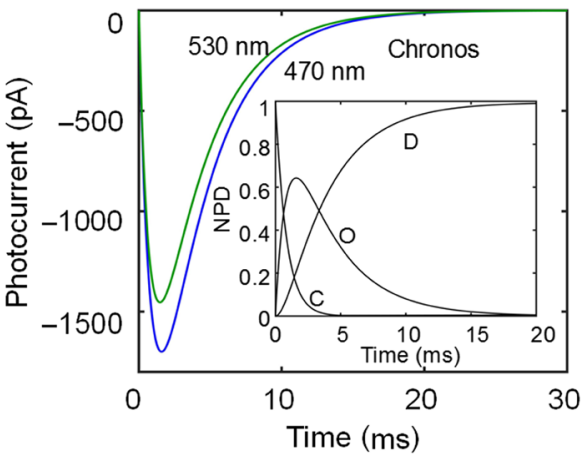

(a)

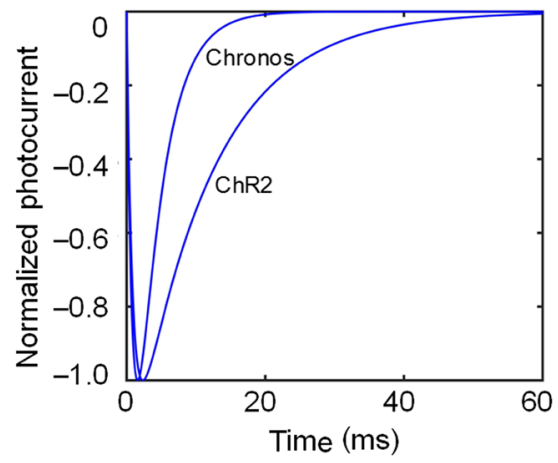

(b)

Fig. 2 (a) Variation of photocurrent of Chronos with time, at 470 and $530 \mathrm{~nm}$, at $I=4.23 \mathrm{~mW} / \mathrm{mm}^{2}$ and $\Delta t=5 \mathrm{~ms}$; inset: corresponding variation of normalized population density with time. (b) Comparison of normalized photocurrent of Chronos and ChR2 at $470 \mathrm{~nm}$.

for a longer period of time due to its large lifetime $(\sim 50 \mathrm{~s})$, before returning back to the initial C-state.

A comparison of the normalized photocurrent of Chronos and ChR2 is shown in Fig. 2(b). Chronos exhibits faster rise and fall times due to its faster kinetics. For $\mathrm{ChR} 2, t_{\text {peak }}$ is $2.35 \mathrm{~ms}$, which is longer than that of Chronos. Theoretical simulations are in excellent agreement with recently reported experimental results. ${ }^{8}$ Under long light irradiance, the current reaches a peak, which decays to a steady-state plateau. The absolute magnitude of the peak and plateau as well as the adaptation ratio (plateau/peak) depends on light irradiance and the total number of ChR2 or Chronos proteins that are illuminated. ${ }^{24-27}$ However, at short time scales, the photocurrent decays back to the baseline, as O-state gets fully depleted, for Chronos $t_{\mathrm{off}} \sim$ $20 \mathrm{~ms}$ and for ChR2 $t_{\text {off }} \sim 60 \mathrm{~ms}$ [Fig. 2(b)].

The effect of irradiance on the photoresponse of Chronos at $470 \mathrm{~nm}$ with pulse width, $\Delta t=5 \mathrm{~ms}$, is shown in Fig. 3(a). $I_{\text {peak }}$ increases with increase in irradiance, as more molecules populate the O-state. A kink appears in the characteristics at lower irradiances, as the number of active channels has not reached the maximum value before light is switched off. Initially, $t_{\text {off }}$ increases with increase in irradiance to a certain value, after which it decreases. The transition to a smooth variation in the photocurrent and the decrease in $t_{\text {off }}$ occur at $I=$ $2 \mathrm{~mW} / \mathrm{mm}^{2}$. The variation of $t_{\text {peak }}$ with irradiance is also shown in Fig. 3(b). It depends on incident light flux. It stays constant from 0.05 to $0.4 \mathrm{~mW} / \mathrm{mm}^{2}$, after which it monotonically decreases and saturates to low values [Fig. 3(b)].

The photocurrrent peaks more rapidly, as desensitization rate is fast and activation and desensitization take place simultaneously. $I_{\text {peak }}$ saturates at higher irradiances as O-state population saturates within the pulse width. The faster buildup of O-state population at higher irradiances leads to a decrease in $t_{\text {peak }}$. A plateau in the photocurrent appears only on continuous excitation, i.e., for pulses of longer duration. A comparison of the variation of the absolute value of $I_{\text {peak }}$ and $I_{\text {plateau }}$ for Chronos and $\mathrm{ChR} 2$ with irradiance for a longer pulse excitation (1 s) is shown in Fig. 4(a). The photocurrent is a result of the cumulative activity of the membrane-resident channel population, which gives rise to $I_{\text {peak }}$, followed by a smaller steadystate $I_{\text {plateau }}$, due to a fraction of the population relaxing to the desensitized D-state. As is evident, $I_{\text {peak }}$ for Chronos is considerably larger than that of ChR2 due to higher conductance and hence higher population of the O-state, whereas $I_{\text {plateau }}$ for Chronos is much smaller than that of ChR2 on account of a lower population remaining in the O-state, since the transition to the D-state is much faster in Chronos.

Interestingly, at very low irradiances, $I_{\text {plateau }}$ rises rapidly and exhibits maxima for Chronos at $I=0.005 \mathrm{~mW} / \mathrm{mm}^{2}$ and, subsequently, increases slightly on increase in irradiance, whereas for ChR2, it rises sharply and saturates to a higher steady-state value [Fig. 4(a)]. The interplay of irradiance-dependent $\tau_{\text {on }}$,

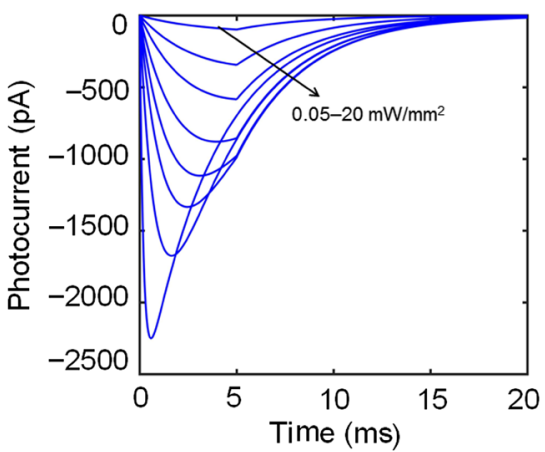

(a)

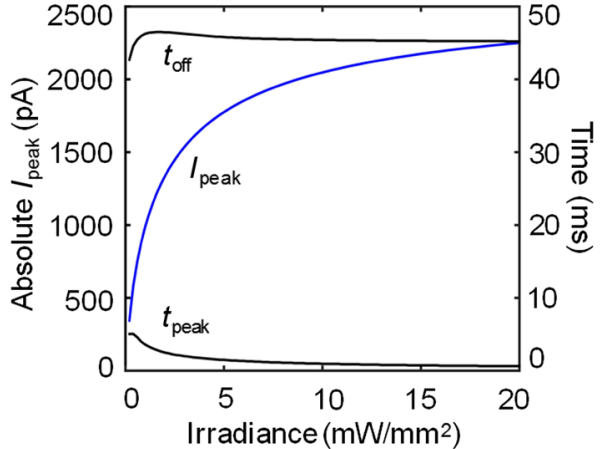

(b)

Fig. 3 (a) Variation of absolute value of peak photocurrent with time of Chronos, at different irradiances $\left(0.05,0.2,0.4,0.8,1.3,2,4\right.$, and $\left.20 \mathrm{~mW} / \mathrm{mm}^{2}\right)$ at $470 \mathrm{~nm}$ and $\Delta t=5 \mathrm{~ms}$. (b) Corresponding variation peak photocurrent, time to peak, and switch-off time with irradiance. 


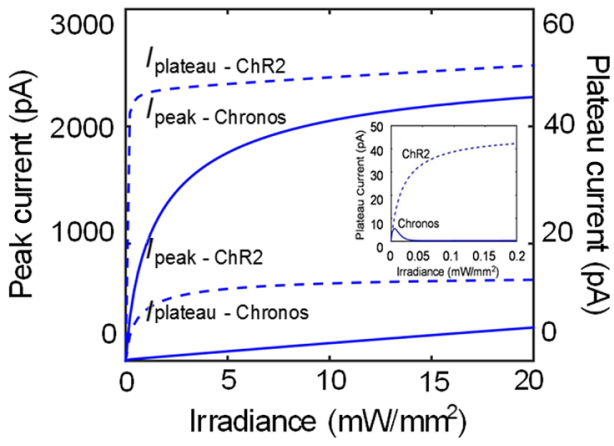

(a)

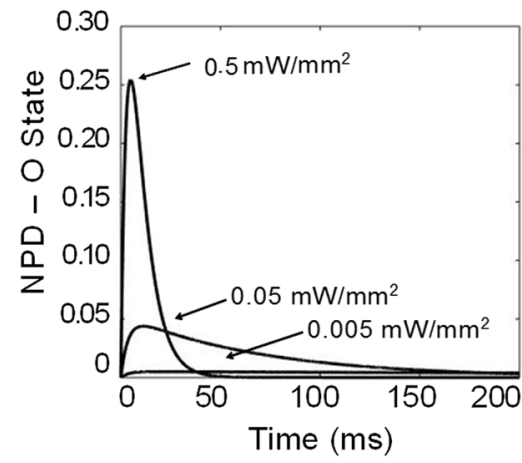

(b)

Fig. 4 (a) Comparison of the variation of peak and plateau currents with time for Chronos and ChR2, at $470 \mathrm{~nm}$ and $\Delta t=1 \mathrm{~s}$; inset shows a magnified view of the variation of plateau current for Chronos and ChR2 at low irradiance values. (b) Corresponding variation of normalized population density of O-state with time.

$t_{\text {peak }}$, and $t_{\text {off }}$ results in the O-state having nearly a constant population at $I=0.005 \mathrm{~mW} / \mathrm{mm}^{2}$ [Fig. 4(b)]. Although, the Ostate population increases with irradiance, it gets rapidly depleted resulting in fall in $I_{\text {plateau }}$ between $I=0.05$ and $0.5 \mathrm{~mW} / \mathrm{mm}^{2}$, as shown in Fig. 4(b). The effect of pulse width on the photocurrent response of Chronos is shown in Fig. 5(a).

For short pulses ranging from 0.5 to $5 \mathrm{~ms}$, increase in the pulse width leads to increase in $I_{\text {peak }}, t_{\text {peak }}$, and $t_{\text {off }}$, which converge after $1.5 \mathrm{~ms}$. The corresponding variations in $I_{\text {peak }}$ and $t_{\text {peak }}$ with pulse width are explicitly shown in Fig. 5(b). As mentioned earlier, the plateau in the photocurrent becomes more prominent for longer pulses and saturates after a certain pulse width. For Chronos, it is at much larger values $(\sim 700 \mathrm{~ms})$ compared to $\mathrm{ChR} 2(\sim 350 \mathrm{~ms})$. $I_{\text {plateau }}$ is also much smaller in Chronos $(-0.22 \mathrm{pA})$ compared to $\mathrm{ChR} 2(-33 \mathrm{pA})$, as shown in Figs. 5(c) and 5(d). A comparison of photocurrents of Chronos and ChR2 for multiple pulse excitation at varying irradiances is shown in Fig. 6. Since the opsin is unable to complete its photocycle before the arrival of the next light pulse, the photocurrent falls to a lower value compared to the first excitation pulse.

At higher irradiances, $I_{\text {peak }}$ saturates as shown in Fig. 3(b). The modulation depends on irradiance, pulse width, and

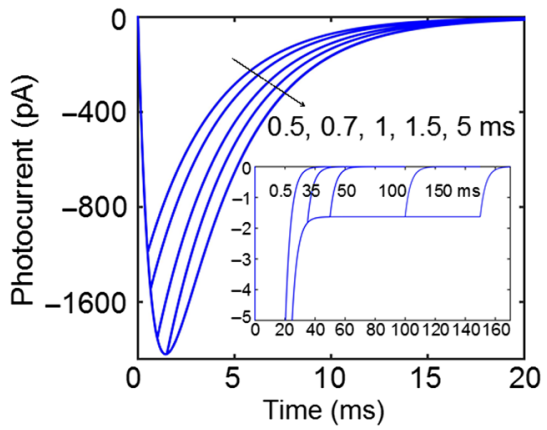

(a)

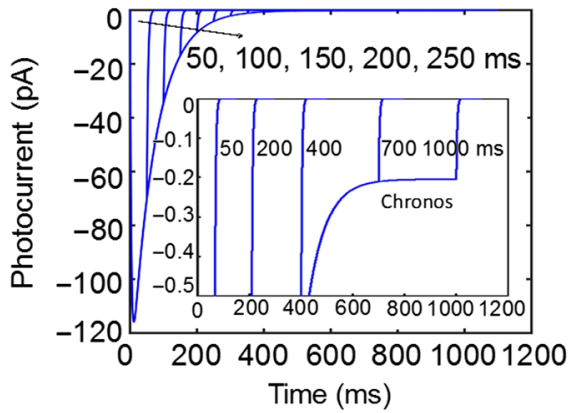

(c)

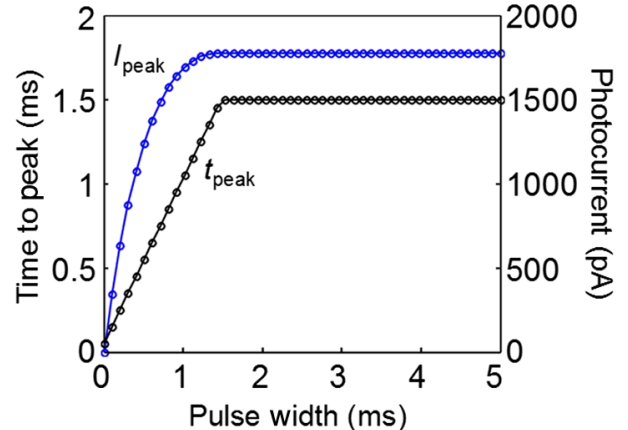

(b)

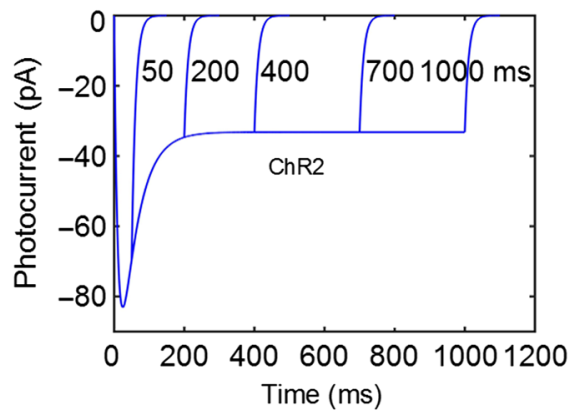

(d)

Fig. 5 (a) Variation of photocurrent with time at different pulse widths, at $I=5 \mathrm{~mW} / \mathrm{mm}^{2}$ at $470 \mathrm{~nm}$; inset: magnified view of photocurrent showing plateau current at different pulse widths. (b) Corresponding variation of time to peak and peak photocurrent with pulse width. (c) Variation of photocurrent at longer pulse widths for Chronos at $I=0.05 \mathrm{~mW} / \mathrm{mm}^{2}$ at $470 \mathrm{~nm}$; inset: magnified view showing the variation of $I_{\text {plateau. }}$ (d) Corresponding variation in $\mathrm{ChR} 2$. 


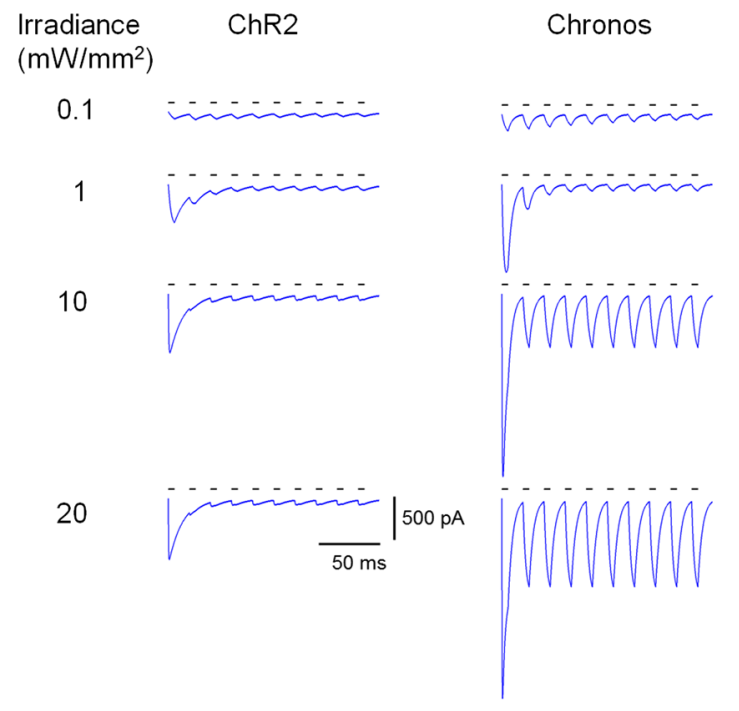

Fig. 6 Comparison of ChR2 and Chronos photocurrents at optostimulation protocol of 10 stimuli of $5-\mathrm{ms}$ pulse width, at $60 \mathrm{~Hz}$, at $470 \mathrm{~nm}$ at indicated irradiances.

frequency of incident optical excitation. Theoretical simulations show that the ratio $I_{\text {peak } 1} / I_{\text {peak } 5}$ also exhibits a maximum with increase in irradiance. The optimal value of irradiance is $1 \mathrm{~mW} / \mathrm{mm}^{2}$, at which this ratio has a maximum value of 13.17. The comparison highlights important differences with Chronos. It is evident that the photocurrent in Chronos is much larger and increases much faster than that in $\mathrm{ChR} 2$, for both primary and secondary peaks, over a wide range of irradiance. The modulation is appreciable in Chronos even at very low irradiances $\left(0.1 \mathrm{~mW} / \mathrm{mm}^{2}\right)$, due to its higher sensitivity. $I_{\text {peak }}$ increases with increase in irradiance and saturates at $20 \mathrm{~mW} / \mathrm{mm}^{2}$ for both Chronos and ChR2. The difference between $I_{\text {peak }}$ and secondary peaks also follows the same pattern.

A comparison of the variation of peak photocurrent with irradiance for multiple light pulse stimulation for Chronos and ChR2 is shown in Fig. 7, for the same protocol as in Fig. 6.

It is evident that the peak is much larger for Chronos and increases at much faster rate with increase in irradiance and saturates at high values. At $5 \mathrm{~mW} / \mathrm{mm}^{2}, I_{\text {peak }}$ for Chronos is $1781 \mathrm{pA}$ and for $\mathrm{ChR} 2$ is $614 \mathrm{pA}$. These values lie near the reported experimental values of 1440 and $800 \mathrm{pA}$, respectively, within indicated error. ${ }^{8}$ Theoretical simulations on

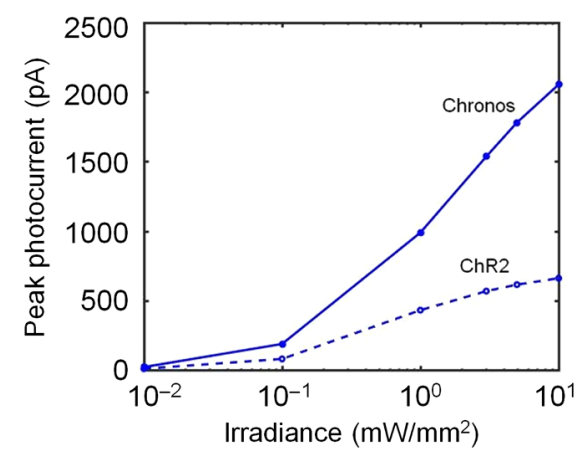

Fig. 7 Comparison of variation in peak photocurrents with irradiance for ChR2 and Chronos at an optostimulation protocol of 10 stimuli each of 5-ms pulse width at $470 \mathrm{~nm}$.

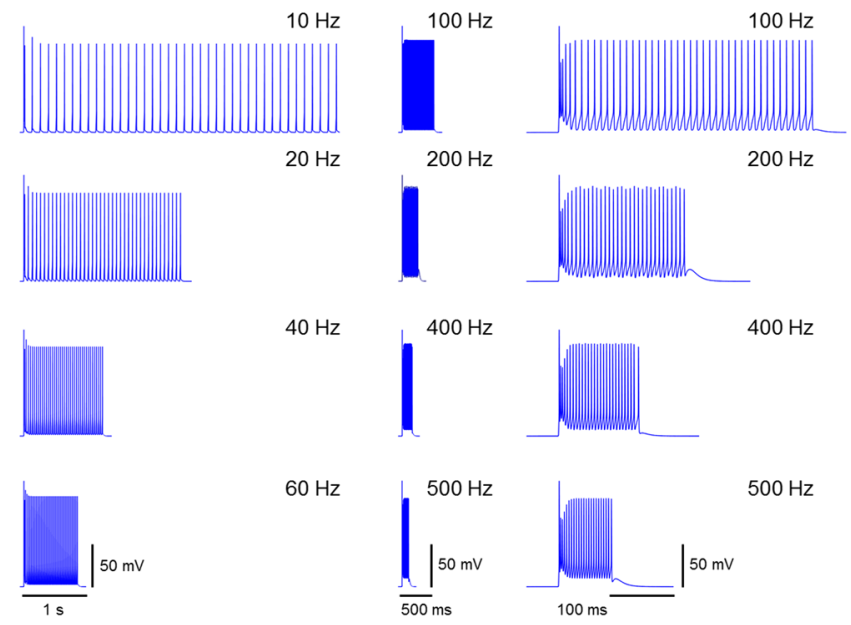

(a)

(b)

Fig. 8 (a) Optical spiking in Chronos-expressing neurons for an optostimulation protocol comprising 40 stimuli each of 2-ms pulse width at $470 \mathrm{~nm}$, at indicated light pulse frequencies, at $5 \mathrm{~mW} / \mathrm{mm}^{2}$, for overall conductance $g_{0}=1 \mathrm{mS} \mathrm{cm}^{-2}$. (b) Magnified view of the variation at higher frequencies.

Chronos-mediated blue-light-driven neuronal spiking fidelity, based on reported experimental parameters, using Eqs. (1)(5) and Tables 1-3, over a wide range of frequencies is shown in Fig. 8. It is interesting to note that detectable spikes are generated even at a frequency as high as $500 \mathrm{~Hz}$, although the spiking fidelity decreases at higher frequencies, as also observed experimentally. ${ }^{8,11}$ The plateau potential also increases on increase in frequency.

Since membrane voltage depends on the light-controlled channel current $I_{\text {Chronos }}$ [Eq. (5)], the variation in it for multiple pulse optostimulation (Fig. 6) results in the corresponding variation in neural spiking as shown in Fig. 8, in which after initial few spikes, the spike modulation decreases and becomes constant. The variation is similar for optostimulation at $530 \mathrm{~nm}^{8}$ Theoretical results capture all the features observed experimentally ${ }^{8}$ at $I=5 \mathrm{~mW} / \mathrm{mm}^{2}$. The effect of blue irradiance on $\mathrm{ChR} 2$ and Chronos spike fidelity corresponding to reported experimental results is shown in Fig. 9.

\section{Irradiance ChR2 $\left(\mathrm{mW} / \mathrm{mm}^{2}\right)$}

0.1

1

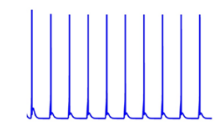

20

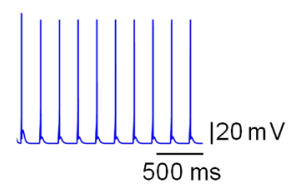

\section{Chronos}
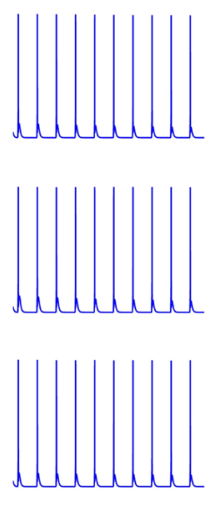

Fig. 9 Comparison of ChR2 and Chronos spike fidelity for optostimulation protocol of 10 stimuli of $5-\mathrm{ms}$ pulse width at $470 \mathrm{~nm}$. The overall conductance $g_{0}=0.09$ and $14.6 \mathrm{mS} \mathrm{cm}^{-2}$ for $\mathrm{ChR} 2$ and Chronos, respectively. 


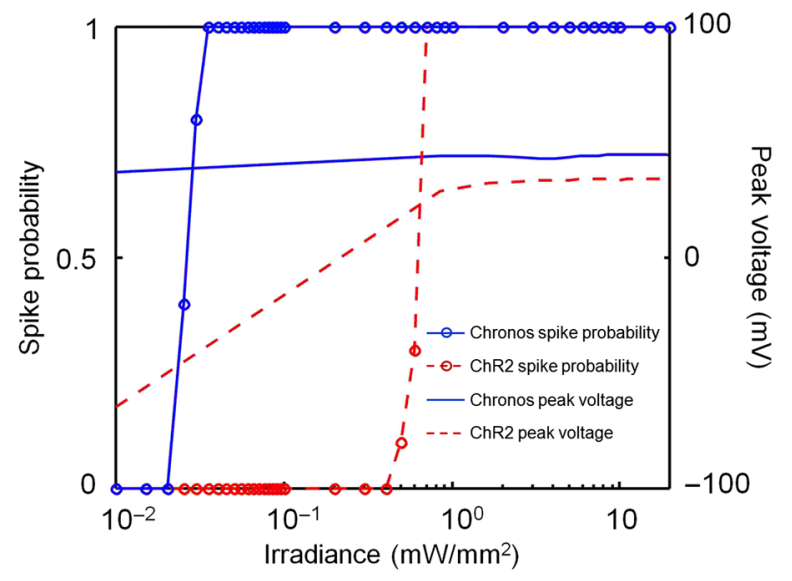

Fig. 10 Comparison of spike probability and peak voltage for optostimulation protocol of 10 stimuli of 5 -ms pulse width at $470 \mathrm{~nm}$, for ChR2 (dashed lines) and Chronos (solid lines), for a particular expression level. The overall conductance $g_{0}=0.09$ and $14.6 \mathrm{mS} \mathrm{cm}^{-2}$ for ChR2 and Chronos, respectively.

There are no spikes with ChR2 at $0.1 \mathrm{~mW} / \mathrm{mm}^{2}$, whereas Chronos exhibits $100 \%$ spiking at this value. The modulation increases with increase in irradiance and eventually saturates for ChR2, while for Chronos, it is high and constant in the entire range. The experimental values of spike height for Chronos at 1 and $20 \mathrm{~mW} / \mathrm{mm}^{2}$ have been reported to be 136 and $141 \mathrm{mV}$, respectively, ${ }^{8}$ while theoretical simulations result in 115 and $117 \mathrm{mV}$, respectively. The effect of irradiance on the spike probability and action potential for Chronos and ChR2, corresponding to Fig. 9, is shown in Fig. 10, for three logs of blue irradiance, for a particular expression level. As is evident from Fig. 10 as well, the spike probability with Chronos is higher at much lower irradiances in comparison to ChR2. Moreover, while the peak voltage for ChR2 increases and saturates at higher irradiances, it is higher and nearly a constant for Chronos in the entire range.

The spike threshold for Chronos and ChR2 has been experimentally reported to be 0.03 and $0.25 \mathrm{~mW} / \mathrm{mm}^{2}$, respectively, while theoretical simulations result in 0.035 and $0.7 \mathrm{~mW} / \mathrm{mm}^{2}$, respectively. ${ }^{8}$ The spike fidelity and the spike probability for Chronos and ChR2, as shown in Figs. 9 and 10, are again in good agreement with recently reported experimental results. ${ }^{8}$ It is known that extra spikes appear in the neural response. ${ }^{20}$ The height, number, and their duration depend on the channel conductance, expression level, optostimulation irradiance, pulse width, and frequency. The effect of conductance along with pulse width on Chronos action potential and extra spiking is explicitly shown in Fig. 11. It can be observed that the action and plateau potentials and number of spikes (with successive spikes decreasing in potential) increase at higher values of conductance and longer pulse width, even at very low value of irradiance. The effect of irradiance and pulse width is similar. The number of spikes increases on increase in both irradiance and pulse width. Elimination of extra spikes for smaller pulse widths, at lower conductance and irradiance values, suits the requirement for energy-efficient ultrafast operation of Chronos. Theoretical simulations provide insights to the optimal values needed to eliminate both extra spikes and plateau potential, to achieve higher precision.

It has also been experimentally demonstrated that the temporal pattern of firing depends on pulse rate. ${ }^{11}$ Although the

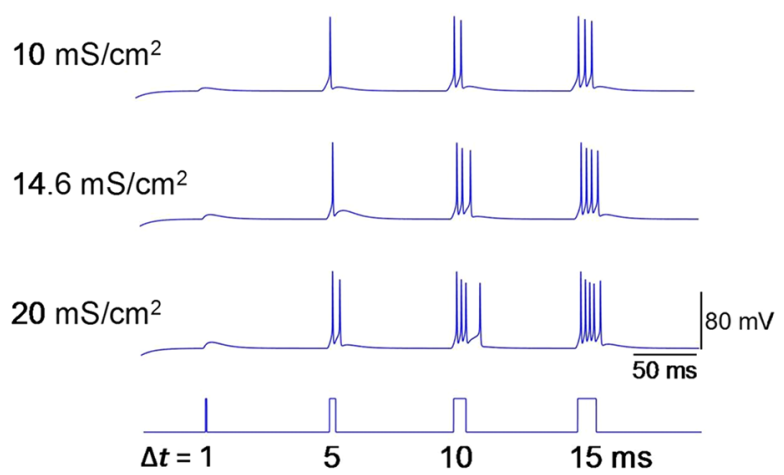

Fig. 11 Effect of increase in conductance and pulse width on Chronos action potential and number of spikes at $470 \mathrm{~nm}$, for $I=0.05 \mathrm{~mW} / \mathrm{mm}^{2}$.

average synchronized index (SI) for both ChR2 and Chronos decreases with increasing pulse rate, Chronos mice exhibit higher SI than ChR2 mice. The driven rates for both were found to be significant over a wide range of stimulus rates ranging from 14 pulses/s to as high as 448 pulses/s. ${ }^{11}$ Chronos exhibits higher driven rate than ChR2 over this entire range. The theoretical variation of driven rate with stimulus rate is as shown in Fig. 12. Chronos exhibits much larger average maximal driven rate of over 200 spikes around 100 pulses/s, each of $1 \mathrm{~ms}$ pulse width, in the intensity range 0 to $200 \mathrm{~mW} / \mathrm{mm}^{2}$.

The variation is in good agreement with the reported experimental variation, within average \pm standard error. There is a maximum driven rate at a particular stimulus rate. The maximum driven rate for $\mathrm{ChR} 2$ is 82 spikes/s in response to 28 pulses/s stimuli, whereas, for Chronos it is 109 spikes/s at the same stimulus rate. The maximum driven rate for Chronos is 277 spikes/s in response to 110 pulses/s stimuli. Average neuronal firing rate over the range of stimulus rate of 14 to 448 pulses/s and intensity range 0 to $200 \mathrm{~mW} / \mathrm{mm}^{2}$ for ChR2 is 43 spikes/s and for Chronos is 128 spikes/s, which is in good agreement with the corresponding reported experimental average rate of 55 spikes/s for ChR2 and 127 spikes/s for Chronos. ${ }^{11}$

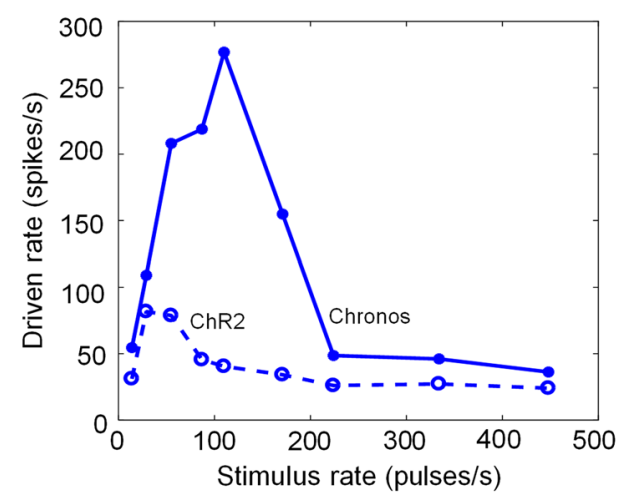

Fig. 12 Variation of average neuronal-driven rate with stimulus rate, with blue light pulses at $473 \mathrm{~nm}$, each of 1 -ms pulse width, at 14 to 448 pulses $/ \mathrm{s}$, in the intensity range 0 to $200 \mathrm{~mW} / \mathrm{mm}^{2}$, for train duration of $500 \mathrm{~ms}$, at $g_{0}=0.85$ and $5.65 \mathrm{~ms} \mathrm{~cm}^{-2}$ for Chronos and ChR2, respectively. 


\section{Discussion}

The excellent agreement of the theoretical simulations with recently reported experimental results highlights the accuracy of the proposed three-state model of the Chronos photocycle and the fast interneuron circuit model for Chronos-expressing neurons. In addition, computational modeling also provides further insights. At $0.005 \mathrm{~mW} / \mathrm{mm}^{2}, I_{\text {plateau }}$ in Chronos has a maximum value of $5.1 \mathrm{pA}$ [Fig. 4(a)]. Optostimulation at $470 \mathrm{~nm}$ with 1.5 -ms pulse-width light pulse results in maximum value of $I_{\text {peak }}$, which saturates on increasing the pulse width beyond this value [Figs. 5(a) and 5(b)]. This is an optimum value for higher temporal resolution. There also exists an optimum value of irradiance of $1 \mathrm{~mW} / \mathrm{mm}^{2}$, at which the ratio $I_{\text {peak } 1} / I_{\text {peak5 }}(=13.17)$ in Chronos exhibits maximum modulation (Fig. 6).

The photoresponse effectively depends on the O-state dynamics that is affected by the parameters studied. The smaller $I_{\text {plateau }}$ in Chronos enables better spiking fidelity. It appears for frequencies higher than $20 \mathrm{~Hz}$ and does not appear at low irradiances even at higher frequencies (Figs. 7 and 8). Chronosexpressing neurons evoke neuronal spikes even at low irradiance and smaller pulse widths. It displays marked temporal stationarity of efficacy over a wide frequency and irradiance range $(0.01$ to $20 \mathrm{~mW} / \mathrm{mm}^{2}$ ) (Fig. 10). Theoretical simulation for ChR2 spike fidelity for the optostimulation protocol in (Fig. 9) does not exhibit plateau potential, although it is observed experimentally. ${ }^{8}$ This could be due to the application of the fast-interneuron $\mathrm{W}-\mathrm{B}$ model used in the theoretical analysis. This feature appears on the application of the $\mathrm{H}-\mathrm{H}$ model to ChR2, which however is unable to capture the details of neuronal spiking in Chronos. The measured temporal properties improve when the opsins are expressed in neurons with faster dynamics. It has been recently shown that transfecting auditory brainstem neurons in mice with ChR2 and Chronos and recording in the inferior colliculus, a strong excitation occurs to 1-ms laser pulses at $473 \mathrm{~nm}$ is much higher with Chronos, upto a stimulation rate of $448 \mathrm{~Hz}$ with Chronos. ${ }^{11}$ The study shows that Chronos has the ability to drive the auditory system at higher stimulation rates than ChR2 and may be a more ideal opsin for manipulation of auditory pathways in future optogenetics-based neuroprostheses. ${ }^{11}$

Chronos reliably drives $100 \%$ spiking at irradiances as low as $0.05 \mathrm{~mW} / \mathrm{mm}^{2}$ and maintains this fidelity over 2 orders of magnitude to $20 \mathrm{~mW} / \mathrm{mm}^{2}$ (Figs. 9 and 10). The fact that Chronos exhibits a lower minimum irradiance threshold compared to ChR2, to achieve $100 \%$ spiking (MIT100), implies that its high effective light sensitivity is not due to higher expression. It can consistently mediate light-sensitive control of neural spiking across a range of expression levels without altering neural excitability. ${ }^{8}$ The response in Fig. 10 has been shown for a particular expression level. However, theoretical simulations show that on increasing the expression levels, the blue irradiance spiking threshold shifts to lower values, as shown in experiments. ${ }^{8}$ It has also been recently shown that efficient current integration enabled by two-photon holographic amplified laser illumination of Chronos evokes spikes in pyramidal cells and interneurons, with sub-ms precision and repeated firing up to $100 \mathrm{~Hz}$ in interneurons. ${ }^{23}$ Since Chronos does not exhibit extra spikes at low intensities and higher driven rates, it is well suited for low-power ultrafast operation.

It is necessary to keep irradiance low to avoid heating effects in the brain. Strong light absorption of blood may be responsible for equal heating for blue and green light. Although scattering is more for blue light, the local absorption minimum of hemoglobin around $480 \mathrm{~nm}$ results in increased absorption of green 532-nm light, which compensates for its lesser scattering. The negligible heating caused by red light highlights the need for red-sensitive opsins to reduce the risk of thermal damage, while providing better depth penetration. ${ }^{34}$ However, the ultrasensitivity of Chronos with spiking at very low irradiances, $\sim 0.05 \mathrm{~mW} / \mathrm{mm}^{2}$ (Fig. 11), enhances the utility of stimulation with blue or green light.

Many neural phenomena that include neural plasticity, behavior, pathology, setting the direction of synaptic change in spike-timing-dependent plasticity, mediating neural coding in systems, such as the auditory system, Parkinson's disease, and psychiatric illness, have been linked to precise spike timing and high-frequency neural events. ${ }^{23}$ It has been shown recently that Chronos-mediated spike fidelity surpasses that of ChR2 and natural acoustic stimulation, to support a superior mid-brain encoding of temporal pulse trains, in terms of firing rate adaptation, temporal coding, and the overall salience of neural responses at high stimulation rates, in central auditory pathway. ${ }^{10}$ These experiments not only demonstrate the temporal coding benefits with new engineered opsins but also highlight the challenge of inducing variegated patterns of forebrain spiking that support adaptive perception and behavior. ${ }^{10}$ In a more recent study, a comparison of ChR-evoked patterns of multiunit activity and local field potentials in primary visual cortex of mice expressing Chronos, Chrimson, and ChR2 was undertaken. ${ }^{35}$ Although all ChR variants caused light-evoked increase in firing in vivo, each demonstrated different temporal patterning of evoked activity and distinct effects on cortical gamma-band activity. Hence, variations in kinetics of optogenetic tools can substantially affect their efficacy in neural networks in vivo, as well as the manner in which their activation engages circuit resonance. ${ }^{35}$

Optogenetic tools also provide the opportunity to address the fundamental question of how important temporal codes are to information processing in the brain. Faster ChRs, such as Chronos, that exhibit higher switching frequencies may suffice for testing most temporal codes, although more information about interactions between different brain areas is required to realize the full potential. ${ }^{36}$ Among other challenges, it is also important to address how large number of neurons can be physically stimulated. The translation of therapeutic or scientific network stimulation protocols to three-dimensional light pattern involves modeling or empirical solutions. A reconfigurable silicon neural processor for real-time simulation and prediction of opto-neural behavior and an integrated circuit for simultaneous extracellular electrophysiology recording and optogenetic neural manipulation have also been recently reported. ${ }^{37,38}$ The theoretical analysis presented here would also be, in general, useful to develop such hardware for optogenetic applications.

\section{Conclusion}

A detailed theoretical analysis of optical control of firing of Chronos-expressing neurons has been presented. A three-state model for Chronos photocycle has been formulated and incorporated in a fast-spiking interneuron circuit model to study optically controlled neural spiking. A detailed study of the effect of various physical parameters, namely, irradiance, pulse width, wavelength, and pulse frequency, on the photoresponse of Chronos and Chronos-expressing neurons has been presented. Theoretical results have been shown to be in excellent agreement with recently reported experimental results. There are 
additional interesting features that are exhibited by Chronos in comparison to ChR2. Optimal values of parameters have been determined for efficient performance. Accurate computational modeling of these neural synaptic events opens up prospects for not only engineering opsins with optimized properties but also understanding and performing precision optogenetic experiments with the desired spatiotemporal resolution.

\section{Disclosures}

No conflicts of interest, financial or otherwise, are declared by the authors.

\section{Acknowledgments}

The authors are grateful to the reviewers for valuable comments and suggestions. They are also grateful to Professor P. S. Satsangi for his kind inspiration, encouragement, and introduction to optogenetics.

\section{References}

1. E. S. Boyden et al., "Millisecond-timescale, genetically targeted optical control of neural activity," Nat. Neurosci. 8, 1263-1268 (2005).

2. K. Deisseroth, "Optogenetics: 10 years of microbial opsins in neuroscience," Nat. Neurosci. 18(9), 1213-1225 (2015).

3. R. D. Airan et al., "Temporally precise in vivo control of intracellular signalling," Nature 458, 1025-1029 (2009).

4. W. K. A. Karunarathne et al., "Subcellular optogenetics-controlling signaling and single-cell behaviour," J. Cell Sci. 128, 15-25 (2015).

5. R. Chen, A. Canales, and P. Anikeeva, "Neural recording and modulation technologies," Nat. Rev. Mater. 2, 16093 (2017).

6. M. Wang et al., "Engineering synthetic optogenetic networks for biomedical applications," Quant. Biol. 5(2), 111-123 (2017).

7. C. M. Ambrosi et al., "Cardiac applications of optogenetics," Prog. Biophys. Mol. Biol. 115, 294-304 (2014).

8. N. C. Klapoetke et al., "Independent optical excitation of distinct neural populations," Nat. Methods 11, 338-346 (2014).

9. J. M. Barrett, R. B. Palmini, and P. Degenaar, "Optogenetic approaches to retinal prosthesis," Visual Neurosci. 31, 345-354 (2014).

10. W. Guo et al., "Hearing the light: neural and perceptual encoding of optogenetic stimulation in the central auditory pathway," Sci. Rep. 5, 10319 (2015).

11. A. E. Hight et al., "Superior temporal resolution of Chronos versus channelrhodopsin-2 in an optogenetic model of the auditory brainstem implant," Hear. Res. 322, 235-241 (2015).

12. N. P. Young and K. Deisseroth, "Cognitive neuroscience: in search of lost time," Nature 542, 173-174 (2017).

13. C. K. Kim, A. Adhikari, and K. Deisseroth, "Integration of optogenetics with complementary methodologies in systems neuroscience," Nat. Rev. Neurosci. 18(4), 222-235 (2017).

14. E. Ferenczi and K. Deisseroth, "Illuminating next-generation brain therapies," Nat. Neurosci. 19(3), 414-416 (2016).

15. E. G. Govorunova et al., "Microbial rhodopsins: diversity, mechanisms, and optogenetic applications," Ann. Rev. Biochem. 86, 845-872 (2017).

16. V. Emiliani et al., "All-optical interrogation of neural circuits," $J$. Neurosci. 35, 13917-13926 (2015).

17. A. Guru et al., "Making sense of optogenetics," Int. J. Neuropsychopharmacol. 18, pyv079 (2015).

18. F. Schneider, C. Grimm, and P. Hegemann, "Biophysics of channelrhodopsin," Ann. Rev. Biophys. 44, 167-186 (2015).

19. R. S. McIsaac, C. N. Bedrbrook, and F. H. Arnold, "Recent advances in engineering microbial rhodopsins for optogenetics," Curr. Opin. Struct. Biol. 33, 8-15 (2015).

20. L. A. Gunaydin et al., "Ultrafast optogenetic control," Nat. Neurosci. 13, 387-392 (2010).

21. T. Mager et al., "Improved microbial rhodopsins for ultrafast red-shifted optogenetics," Biophys. J. 114(3), p669a (2018).

22. E. G. Govorunova et al., "The expanding family of natural anion channelrhodopsins reveals large variations in kinetics, conductance, and spectral sensitivity," Sci. Rep. 7, 43358 (2017).
23. E. Ronzitti et al., "Sub-millisecond optogenetic control of neuronal firing with two-photon holographic photoactivation of Chronos," J. Neurosci. 37(44), 10679-10689 (2017).

24. K. Nikolic et al., "Photocycles of channelrhodopsin-2," Photochem. Photobiol. 85, 400-411 (2009).

25. N. Grossman et al., "Modeling study of the light stimulation of a neuron cell with channelrhodopsin-2 mutants," IEEE Trans. Biomed. Eng. 58, 1742-1751 (2011).

26. R. Stefanescu et al., "Computational modeling of channelrhodopsin-2 photocurrent characteristics in relation to neural signaling," Bull. Math. Biol. 75, 2208-2240 (2013).

27. N. Grossman et al., "The spatial pattern of light determines the kinetics and modulates backpropagation of optogenetic action potentials," J. Comput. Neurosci. 34, 477-488 (2013).

28. B. D. Evans et al., "PyRhO: a multiscale optogenetics simulation platform," Front. Neuroinf. 10, 1-19 (2016).

29. S. Roy, C. P. Singh, and K. P. J. Reddy, "Generalized model for alloptical light modulation in bacteriorhodopsin," J. Appl. Phys. 90, 3679-3688 (2001).

30. S. Roy et al., "All-optical switching in pharaonic phoborhodopsin protein molecules," IEEE Trans. Nanobiosci. 5, 178-187 (2006).

31. S. Roy and C. Yadav, "All-optical sub-ps switching and parallel logic gates with bacteriorhodopsin (BR) protein and BR-gold nanoparticles," Laser Phys. Lett. 11, 125901 (2014).

32. A. L. Hodgkin and A. F. Huxley, "A quantitative description of membrane current and its application to conduction and excitation in nerve," J. Physiol. 117, 500-544 (1952).

33. X. J. Wang and G. Buzsáki, "Gamma oscillation by synaptic inhibition in a hippocampal interneuronal network model," J. Neurosci. 16, 64026413 (1996).

34. G. Arias-Gil et al., "Measurement, modeling, and prediction of temperature rise due to optogenetic brain stimulation," Neurophotonics 3, 045007 (2016).

35. N. Y. Jun and J. A. Cardin, "Channelrhodopsin variants engage distinct patterns of network activity," bioRxiv 275644 (2018).

36. G. Baranauskas, "Can optogenetic tools determine the importance of temporal codes to sensory information processing in the brain?" Front. Syst. Neurosci. 9, 174 (2015).

37. J. Luo et al., "Optogenetics in silicon: a neural processor for predicting optically active neural networks," IEEE Trans. Biomed. Cir. Syst. 11, 15-27 (2017).

38. C. H. Chen et al., "An integrated circuit for simultaneous extracellular electrophysiology recording and optogenetic neural manipulation," IEEE Trans. Biomed. Eng. 64, 557-568 (2017).

Sant Saran received his BTech degree in electrical engineering from Dayalbagh Educational Institute, India, in 2017. His project on optogenetic control of neuronal signaling was awarded the Best Innovative Student Project Award by the Indian National Academy of Engineering in 2017. He was also awarded the Best Poster Award at the IEEE Seminar on Biometric Recognition Systems organized by Michigan State University in 2015. Currently, he is a project validation engineer at Cadence Design Systems, Noida, India.

Neha Gupta is pursuing her PhD in the Department of Physics and Computer Science, Dayalbagh Educational Institute, India. She received her MSc degree in physics from Kumaon University and MTech degree in solid-state electronics from the Indian Institute of Technology Roorkee in 2007 and 2011, respectively. She has taught at Teerthanker Mahaveer University, Moradabad, and in colleges affiliated to Maharishi Dayanand University, Rohtak, and Indraprastha University, Delhi, India. Her research interest is focused on computational modeling of optogenetic neuronal switching.

Sukhdev Roy is a professor in the Department of Physics and Computer Science, Dayalbagh Educational Institute, India. He received his $\mathrm{PhD}$ from the Indian Institute of Technology Delhi in 1993 and has been a visiting scientist at Harvard University. $\mathrm{He}$ is a fellow of the National Academy of Sciences, India and an associate editor of IEEE Access. His research interests are in quantum-, nano-, and biophotonics, especially all-optical ultrafast information processing with natural photoreceptors and optogenetically controlled neurons. 